\title{
The MIRAX mission: Payload description and background simulation plan
}

\author{
Manuel Castro* \\ Instituto Nacional de Pesquisas Espaciais (INPE), São José dos Campos, SP, Brazil. \\ E-mail:castroddas.inpe.br \\ João Braga \\ Instituto Nacional de Pesquisas Espaciais, São José dos Campos, SP, Brazil. \\ E-mail:[ioao.braqadinpe.br
}

\section{Flavio D'Amico}

Instituto Nacional de Pesquisas Espaciais, São José dos Campos, SP, Brazil.

E-mail:damicodas.inpe.br

\section{Josh Grindlay}

Harvard Smithsonian Center for Astrophysics, Cambridge, MA, USA.

E-mail:ioshehead.cfa.harvard.edu

\section{Richard Rothschild}

University of California San Diego, San Diego, CA, USA.

E-mail: rrothschildaucsd.edu

MIRAX (Monitor e Imageador de RAios X) will be the first Brazilian astronomical space mission. It will image the sky from 5 to $200 \mathrm{keV}$ to perform timing and spectral observations of transient sources in a wide field of view $\left(360^{\circ} \times 60^{\circ}\right.$ each 1.5 -hr orbit) with $\sim 1$ arcmin position accuracy. The satellite bus and launch (in 2017) will be provided by INPE/Brazil. The MIRAX payload consists of four coded-mask telescopes in a $2 \times 2$ array. Each telescope has a detector plane made by an array of $8 \times 8$ pixelated CdZnTe (CZT) detectors with $0.6 \mathrm{~mm}$ pitch, provided by the Harvard-Smithsonian Center for Astrophysics. The lateral and lower graded shields are made of lead, tin, copper and aluminum layers. A Tungsten coded mask is placed $70 \mathrm{~cm}$ away from the detector plane. In orbit, the instrument will be exposed to a radiation field mainly composed of photons, electrons, protons and neutrons that interact in the detectors and satellite materials, producing background for astrophysical observations. In this work, we present a description of instrument, its scientific goals and the plan to perform background simulations with the Geant4 package using the MIRAX experimental configuration and the spectral and angular distribution of the background sources. To calculate the background spectra that are used as input in the simulations, we use the SPENVIS (SPace ENVironment Information System) system, which provides the background spectra for each particle for specific altitudes and orbital inclinations.

An INTEGRAL view of the high-energy sky (the first 10 years)" 9th INTEGRAL Workshop and celebration of the 10th anniversary of the launch,

October 15-19, 2012

Bibliotheque Nationale de France, Paris, France 


\section{Introduction}

MIRAX (Monitor e Imageador em RAios X) will be the first Brazilian-led astronomical space mission. It will image the sky from 5 to $200 \mathrm{keV}$ to perform timing and spectral obsevations in a wide field of view $\left(60^{\circ} \times 60^{\circ} \mathrm{FWHM}\right)$ with a $\sim 1$ arcmin position accuracy $(10 \sigma$ source $)$ and spectral resolution of $\sim 2 \mathrm{keV}$. The mission has three primary science objectives:

- Conduct a deep, high resolution survey of the Galactic bulge and plane to measure the black hole vs neutron stars population in the Galaxy. The distinction between the two classes of sources can be made based on spectral characteristics, presence or absence of pulsations and bursts, among other observational properties.

- Study high energy time domain astrophysics of compact objects (accreting neutron stars and black holes) and the physics of their disk and jets on a wide range of scales, from faint transients and thermonuclear bursts on neutron stars to energetic flares from relativistic jets in blazar active galactic nuclei.

- Detect short Gamma-Ray Bursts (sGRBs) with precise locations of 10-30 arcsec and correlate these positions with data from Advanced Ligo gravitational wave observatory.

MIRAX will operate in scanning mode, with the X-ray axis always oriented $25^{\circ}$ South of the zenith direction. In this way, we will maximize the coverage of the Central Galactic plane region in each near-equatorial $\left(15^{\circ}\right)$ circular LEO $(\sim 650 \mathrm{~km})$ orbit of the satellite. In each 96 minute orbit, MIRAX will scan a $360^{\circ} \times 70^{\circ}$ region of the sky up to zero intensity, so in every 54-day precession period of the orbit, MIRAX will scan all the sky between $+30^{\circ}$ and $-80^{\circ}$ in declination. MIRAX will use an X-band telemetry system and will send data every orbit to an equatorial ground station in Alcântara, MA, Brazil. The mission will employ a free-data approach in which all the main data products will be processed as soon as possible and made available immediately to the community at the MIRAX data centers.

Here we present a technical description of MIRAX and the background simulation plan. We developed a very detailed mass-model of all the materials in the experimental configuration, and will perform a set of Monte Carlo Geant4-based simulations to study the instrument behavior at its circular $650 \mathrm{~km}$ altitude near-equatorial orbit. In order to estimate the external particle background spectra at the MIRAX orbit, which are used as input for the simulations, we have used the SPENVIS software avalaible at the Internet.

\section{Technical description}

The MIRAX observatory consists of a $2 \times 2$ array of coded-mask imaging telescopes. Each telescope has a fully coded field of view (FCFOV) of $25^{\circ} \times 25^{\circ}$. The four telescopes are mutually offset to give a combined FCFOV of $50^{\circ} \times 50^{\circ}$ and a half-coded field of view of $60^{\circ} \times 60^{\circ}$. Each telescope is composed of a detector plane, passive shielding layers and a coded mask. The basic detection unit is the Detector Crystal Unit (DCU) made of pixelated CZT(Cadmium Zinc Telluride),

*Speaker. 
a "state of the art" material for hard X-ray and soft $\gamma$-ray detection. A $2 \times 2$ array of DCUs make a Detector Crytal Array (DCA) and a $2 \times 2$ array of DCAs make a Quad Detector Module(QDM). Finally, a $2 \times 2$ array of QDMs constitute a Detection Module (DM). Each shielding layer comprises four sublayers: lead, tin, copper and aluminum, where the lead is the outer layer and the aluminum is the inner layer. The shielding layer can be divided in two parts: A lower section of $43 \mathrm{~cm}$ (vertical distance) and the upper section. $70 \mathrm{~cm}$ away from the detection plane is placed a coded mask. In the figure $\square$, we show an overview of the MIRAX instruments (see table $\square$ for more details).
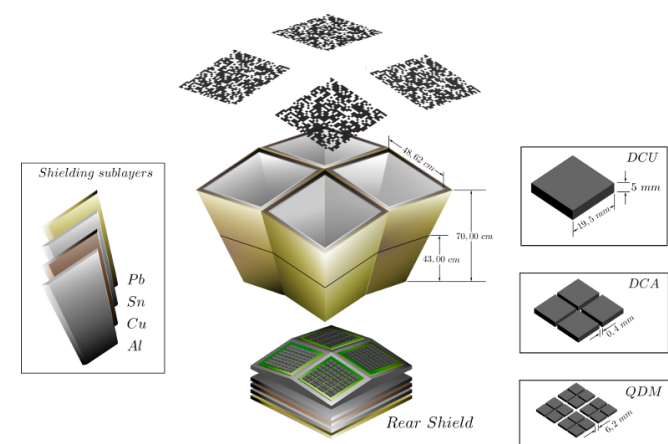

Figure 1: Exploded diagram of the four Hard Xray Imagers of MIRAX.

\begin{tabular}{|c|c|c|}
\hline \multicolumn{3}{|c|}{ Detection Module } \\
\hline & DCU size & $19.5 \times 19.5 \times 5.0 \mathrm{~mm}$ \\
\hline Spatial & esolution of each DCU & $0.6 \mathrm{~mm}$ \\
\hline Gap betw & en close-tiled $2 \times 2$ DCUs & $0.4 \mathrm{~mm}$ \\
\hline & p between DCAs & $6.2 \mathrm{~mm}$ \\
\hline \multicolumn{3}{|c|}{ Shielding sublayer thickness } \\
\hline Sublayer & Lower section (mm) & $\begin{array}{l}\text { Upper section }(\mathrm{mm}) \\
\end{array}$ \\
\hline $\mathrm{Pb}$ & 0.6 & 0.3 \\
\hline $\mathrm{Sn}$ & 0.4 & 0.2 \\
\hline $\mathrm{Cu}$ & 0.1 & 0.1 \\
\hline $\mathrm{Al}$ & 0.25 & 0.12 \\
\hline \multicolumn{3}{|c|}{ Coded Mask } \\
\hline & Pattern & Random \\
\hline & Open fraction & 0.4 \\
\hline & Material & ungsten \\
\hline & Element size & $1.1 \times 0.3 \mathrm{~mm}$ \\
\hline
\end{tabular}

Table 1: Main instrumental features of the MIRAX instrument.

\section{Simulations with GEANT4}

Using the Geant 4 [W] package, a tool developed by CERN to simulate the interaction of particles and radiation with matter, and using the MIRAX geometrical description (the mass model), we are performing a series of simulations to study MIRAX responses and background spectra. We take into account each part of the telescope (detection plane, shielding layers and coded mask) with their composition in terms of materials. We use each material and its dimensions (especially thickness) to program the MIRAX geometry using the Geant 4 classes already available in the package.

Geant 4 requires input spectra of the particles that interact with the mass model. In this application we define the spectral and angular distribution of the particles coming from the space and Earth's atmosphere at the MIRAX's circular orbit of $\sim 650 \mathrm{~km}$ and $15^{\circ}$ inclination. The satellite will be hit by a field of particles composed mainly of protons, neutrons, electrons and photons which will interact with all materials. As a result of these interactions, X-ray photons will be produced and a fraction of those will eventually hit the CZT detectors. Those, in addition to the cosmic X-ray flux coming from the telescope apertures, comprise the background the instrument will measure. Using the SPENVIS (SPace ENVironment Information System) [వ] software we can calculate the spectra for each particle species and use it as a input in Geant4.

Up to now, we have already modeled the full geometry of one single telescope. Currently we are in the process of "rotate" one telescope to obtain $2 \times 2$ array of the instrument. As each telescope 
has a pyramidal shape, when the array is built the four telescopes share the same edge.

Using SPENVIS, we are able to calculate the spectra from three particle fields: trapped particles, solar particles and cosmic rays. First we need to generate the spacecraft trajectory using the orbital parameters (altitude and inclination), the launch date and mission duration. Then we can choose the background source and calculate the fluxes and spectra that are used as input in the background simulations.

\section{Conclusions and perspectives}

- MIRAX (Monitor e Imageador de RAios X) is the first Brazilian-led space mission devoted to astrophysics. MIRAX will carry out an unprecedented wide-band X-ray survey of the Galaxy and is especially suited for high-cadence monitoring of transient sources. The mission will be launched in 2017 for a lifetime of 4 years.

- We have developed a detailed mass model for one of the 4 identical hard X-ray imagers (HXIs) of MIRAX in order to perform Monte Carlo simulations to calculate the instrument response in a near-equatorial circular LEO. We will use the trapped particle and cosmic ray data available at the ESA's SPENVIS website, and also the cosmic diffuse X-ray emission, to use as inputs to a series of Geant4 simulations.

- We are now finalizing the instrument mass model to account for the 4 HXIs in the flight configuration. We are also converting the particle spectra available at SPENVIS into input files for Geant 4 .

\section{References}

[1] Agostinelli, S., Alisson, J., Amako, K., et al., Geant4 - a simulation toolkit, Nucl. Instrum. Methods Phys. Res., Sect. A, 506, 250 (2003).

[2] http://www.spenvis.oma.be/ 